\title{
Personalien und Tagesnachrichten
}

Privatdozent Dr. Oskar Kieß, Oberarzt der Dermatologischen Klinik in Leipzig, wurde zum außerordentlichen Professor ernannt.

Dr. Asia v. Mallinckroät-Haupt in Düsseldorf hat sich für Haut- und Ge-schlechtskrankheiten habilitiert; ihre Hauptarbeiten hat sie aus der Berliner und Bonner Hautklinik veröffentlicht. Privatdozent Dr. Schmidt-La Baume, Oberarzt der Dermatologischen Uni-versitäts-Klinik in Frankfurt a. M., ist zum Letter der Dermatologischen Ab-teilung im Städtischen Krankenhaus Mannheim ernannt und mit der Führung des dortigen Gesundheitsamtes betraut worden. Am 1. September tritt Prof. Arth. Jordan von der Leitung der Zweiten Moskauer Hautklinik zurück. Sein Nachfolger ist Prof. N. Efron.

Die Professoren Hans Meyer in Bremen, Gans in Frankfurt a. M. und Meinicke in Ambrock wurden zu korrespondierenden Mitgliedern der Dänischen Dermatologischen Gesellschaft ernannt, ferner wurde Prof. 0. Gans in Frankfurt a. M. zum Ehrenmitglied der Société Française de Sérologie appliquée sowie der Société Française de la Prophylaxie sanitaire et morale gewählt.

288

Personalien und Tagesnachrichten.

Prof. Dr. Buschke, Leiter der Dermatologischen Abteilung am Rudolf-Virchow-Krankenhaus Berlin, wurde zum Ehrenmitglied der Hellenischen Gesellschaft für Dermatologie Athen gewählt.

Prof. G. Nobl in Wien wurde zum Ehrenmitglied der Japanischen Dermatologischen Gesellschaft gewählt.

Prof. Dr. Johan Almkvist, Direktor der Dermatologischen Universitäts-Klinik in Stockholm, hielt in Hamburg auf Einladung der Medizinischen Fakul-tät einen Vortrag „Aus der Geschichte der Quecksilberschädigungen". Die Fakultät verlieh ihm ihre goldene Ehrenmünze.

Dr. P. Ravaut in Paris wurde zum Präsidenten der Société de Dermatologie et de Syphiligraphie ernannt.

Prof. Dr. Alfred Brandiveiner, außerordentlicher Professor für Dermatologie und Syphilidologie an der Universität Wien und Abteilungsvorstand der Allgemeinen Poliklinik, ist am 21. Juni im 57. Lebensjahr gestorben.

In Mexiko fand am 6. März dieses Jahres für die deutschen Eorscher Ehrlich, Wassermann und Schaudinn eine eindrucksvolle Peier statt. Der Präsident der Republik betonte die Bedeutung der drei Gelehrten, die durch ihre Arbeiten zur Bekämpfung der Syphilis so viel beigetragen haben. Der deutsche Gesandte wohnte der Feier bei.

Auf verschiedene Anfragen, die wegen des Ausfalls der Wiener Tagung an die Schriftleitung gerichtet wurden, kann folgendes gesagt werden. Seitens der Deutschen Dermatologischen Gesellschaft ist über die Zeit der Abhaltung des verschobenen Wiener Kongresses noch keine Entscheidung bekanntgegeben worden. Bis zum vorgeschriebenen Termin haben nur etwa 60 Anmeldungen vorgelegen. Der Umstand, daß für die letzten Kongresse (Bonn 1927, Königs-berg 1929 und nun Wien) immer peripher gelegene Orte gewählt wurden, die eine sehr iveüe und 
teuere Reise für viele erfordern, seheint bei dieser Kon-greß-Onlust eine Rolle zu spielen. Für die Einladung seitens der Stadt und Hochschule Hannover, die in Königsberg vorlag, hätte wohl diese Schwierig-keit nicht bestanden. Vielleicht empfiehlt es sich, bei der Dermatologischen Sektionstagung der Naturforscher- und Ärzteversammlung in Mainz und Wiesbaden Ende September dieses Jahres zu dieser Frage die Meinung der an-wesenden Kollegen zu hören; ebenso bei der Herbsttagung der südwest-deutschen Dermatologen in Frankfurt und der rheinisch-westfälischen in Dortmund, die etwas später stattfinden. Es ware doch sehr erwünscht, wenn eine größere Tagung nicht allzu lange verschoben würde. Hierzu wurde angeregt, die Wiener Tagung, falls sie nicht früher stattfinden kann, kurz vor den internationalen Kongreß in Budapest zu legen, damit beide Veranstaltungen - natürlich gegenseitiges Einvernehmen vorausgesetzt - mit einer einzigen Reise besucht werden können (Herbst 1934). Vorher ware dann vielen wohl eine etwas größere Tagung in MitteldeutschL·nd erwünscht, die, ähnlich wie die Tagungen in der schlimmen Zeit von 1917 und 1918 in Bonn und Berlin, inoffiziell einberufen werden könnte. 